\title{
Décision CPI n 05042 Indemnisation des rapports
}

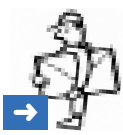

La décision de la CPI concernant l'indemnisation des rapports dans le TARMED est insatisfaisante [1]: La position 00.2250 indemnise un petit rapport, sur formulaire ou non, adressé à l'assureur à sa demande. Personne n'aurait l'idée de le facturer deux fois à l'assureur!

Les positions 00.2270 à 00.2290 sont des rapports rédigés sans formulaires, p. ex. la correspondance entre médecins. Cette activité médicale peut, bien entendu, être facturée au répondant des coûts, mais certainement pas deux fois!

Le problème se trouve toutefois ailleurs, à la position 00.2260 qui n'est pas touchée par la décision de la CPI. Cette position indemnise les copies de rapports existants qui ont déjà été facturés à l'assureur (positions 00.2270 à 90 ) et que l'assureur demande ultérieurement sous forme de copies, générant ainsi un travail supplémentaire (recherche du dossier médical et du rapport / prise de décision d'ordre médical quant à la possibilité légale de transmettre ce rapport à l'assureur pour consultation / copie et envoi du rapport).

Les assureurs (SCTM) défendent le point de vue selon lequel ils ont un droit sur chaque rapport qu'ils indemnisent et que ce droit est gratuit et automatique!

A ma demande, le service tarifaire de la FMH m'a répondu le 4 mai 2004 que cette copie demandée ultérieurement pouvait être facturée avec la position 00.2260. Le chapitre $00.06 \mathrm{du}$ TARMED n'interdit pas les cumuls!

A une question semblable posée par l'assureur, le même service tarifaire de la FMH donne le 27 mai 2004 un renseignement contraire et adopte la position de l'assureur en répondant que les copies demandées ne peuvent plus faire l'objet d'une facturation supplémentaire.

Dans quel but a-t-on créé la position 00.2260 qui note explicitement dans son interprétation médicale qu'en l'occurrence «il s'agit des copies de documents médicaux existants demandées par l'assureur»?
Indépendamment de cela, l'argument de la SCTM est absurde! Quand un assureur indemnise une activité médicale (p.ex. rédaction d'une lettre), pourquoi a-t-il automatiquement un droit sur ce produit de l'activité médicale? Quand il rembourse un médicament, a-t-il aussi un droit sur le médicament? Quand il paie une hospitalisation, peut-il aussi bénéficier du séjour? De surcroît, n'oublions pas les problèmes qui surgiraient en matière de protection des données si chaque échange épistolaire devait automatiquement être transmis à l'assureur!

Dans sa décision, la CPI n'a absolument pas tenu compte de cette controverse!

\section{Dr Martin Schilt, Lucerne}

1 TARMED Suisse. Décisions de la CPI. Bull Méd Suisses 2005;86(48):2661.

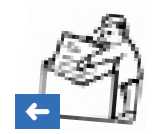

\section{Réplique}

Le chapitre $00.06 \mathrm{du}$ TARMED a été abondamment discuté et mis au point par la Commission paritaire TARMED (CPT) durant l'hiver 2005/ 2006. Les quatre partenaires tarifaires TARMED se sont mis d'accord sur une solution qui a été confirmée par le comité de direction de TARMED. La version remaniée du chapitre 00.06 et de l'interprétation générale 14 (IG-14) le concernant sera publiée dans le nouveau logiciel de navigation TARMED. Mais pour ce faire, nous attendons (depuis plusieurs mois) l'approbation du Conseil fédéral. Voici une information préalable à ce sujet:

\section{IG-14}

Tous les rapports et documents doivent être fournis à l'assureur ou au médecin-conseil de l'assureur à sa demande, dans le respect des dispositions de la protection des données.

Chaque prestation doit être documentée sous la forme appropriée (écrit, imprimé, bande magnétique ou support d'image). 
La documentation et le rapport font partie intégrante de la prestation et sont indemnisés avec celle-ci. Un minutage ne figure pas non plus sous le paramètre «rapport». Le premier envoi des documents à l'assureur est effectué gratuitement, pour autant qu'il l'ait demandé. Demeurent réservées les prestations pour lesquelles une facturation séparée de la documentation ou du rapport est expressément mentionnée et tarifée.

L'envoi répété de ces documents à l'assureur, dans la mesure où il l'a exigé, est facturé avec la position 00.2260 ou 00.2265 .

\section{Commentaire selon le point de vue de la FMH}

Conformément au paragraphe «Médecinconseil», les dispositions relatives à la protection des données doivent être respectées au moment de l'envoi des documents. Lorsque vous les adressez au médecin-conseil, vous devriez avoir rempli ces dispositions. En revanche, vous ne pouvez pas être poursuivi devant les tribunaux pour l'examen interne, par l'assureur, des documents envoyés au médecin-conseil.

Il n'est écrit nulle part que des copies doivent être «automatiquement» envoyées à l'assureur mais uniquement à sa demande.
Conclusion: il faut faire la distinction entre la nécessité d'établir un nouveau rapport pour l'assureur et la copie de documents existants. Lorsque l'assureur demande une nouvelle copie, vous pouvez la facturer avec la position appropriée du TARMED (position $\mathrm{n}^{\circ} 00.2260$ [telle qu'elle existe] ou $\mathrm{n}^{\circ} 00.2265$ [lorsque la copie compte plus de 10 pages]). Si un rapport est établi en supplément, vous pourrez le facturer avec la nouvelle position TARMED no 00.2285 (rapport rédigé sans formulaire, 11 à 35 lignes de texte) et, selon le cas, aussi avec la nouvelle position TARMED 00.2295 (Rapport rédigé sans formulaire, par 35 lignes de texte supplémentaires).

Dernière remarque: un rapport est considéré comme rédigé sans formulaire selon le TARMED lorsqu'une caisse-maladie a créé son propre formulaire. Il est également considéré comme rédigé sans formulaire lorsque son auteur (le médecin) emploie un modèle personnel semblable à un formulaire.

M.-C. Peter, membre du Comité central de la FMH 